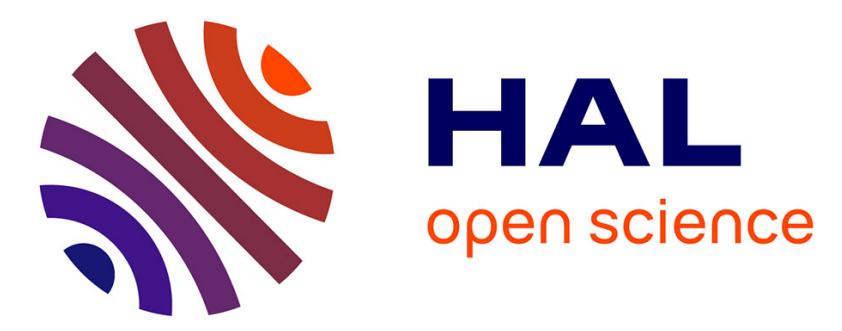

\title{
Spectrally-efficient SIMO relay-aided underlay communications: An exact outage analysis
}

\author{
Zakaria El-Moutaouakkil, Kamel Tourki, Samir Saoudi
}

\section{To cite this version:}

Zakaria El-Moutaouakkil, Kamel Tourki, Samir Saoudi. Spectrally-efficient SIMO relay-aided underlay communications: An exact outage analysis. ICC 2014: IEEE International Conference on Communications, Jun 2014, Sydney, Australia. pp.1531 - 1536, 10.1109/ICC.2014.6883539 . hal01188823

\section{HAL Id: hal-01188823 \\ https://hal.science/hal-01188823}

Submitted on 31 Aug 2015

HAL is a multi-disciplinary open access archive for the deposit and dissemination of scientific research documents, whether they are published or not. The documents may come from teaching and research institutions in France or abroad, or from public or private research centers.
L'archive ouverte pluridisciplinaire HAL, est destinée au dépôt et à la diffusion de documents scientifiques de niveau recherche, publiés ou non, émanant des établissements d'enseignement et de recherche français ou étrangers, des laboratoires publics ou privés. 


\title{
Spectrally-Efficient SIMO Relay-aided Underlay Communications: An Exact Outage Analysis
}

\author{
Zakaria El-Moutaouakkil(1), Kamel Tourki(2), Samir Saoudi(1) \\ (1) Institut TELECOM/TELECOM Bretagne/UMR CNRS 3192 Lab-STICC, Brest, France \\ ${ }^{(2)}$ Electrical \& Computer Engineering Program, Texas A\&M University at Qatar, Doha, Qatar \\ Emails: \{zakaria.elmoutaouakkil, samir.saoudi\}@telecom-bretagne.eu, kamel.tourki@qatar.tamu.edu
}

\begin{abstract}
In this paper, we carry out an exact outage analysis for a secondary (unlicensed) system operating under a strict primary (licensed) system outage constraint. We focus on single-user singleinput multiple-output (SIMO) secondary communications where the direct link is being assisted by a cluster of single-antenna decodeand-forward (DF) relay nodes acting in a half-duplex selective-andincremental relaying mode. Firstly, we derive a transmit power model for the secondary system where the source and relays adapt their transmit power based on: 1) a perfect acquisition of the underlying interference channel state information (I-CSI), and 2) an interference constraint that is either fixed or proportional to the primary system outage probability. Secondly, the cumulative distribution functions (CDF)s of the received signal-to-noise ratio (SNR) at the secondary receiving nodes are devised in a recursive and tractable closed-form expressions. These statistics are used to derive the exact end-to-end secondary system outage probability. The analytical and simulation results are then compared and interestingly shown to perfectly match, while revealing that with a moderate number of primary and secondary receive antennas, the secondary system spectral efficiency is amply enhanced as opposed to being severely degraded in the single receive antenna case.

Index Terms-Underlay, SIMO, decode-and-forward, selectiveand-incremental relaying, outage probability.
\end{abstract}

\section{INTRODUCTION}

In response to the ever-growing stress put on the wireless spectrum medium, cognitive radio (CR) has recently emerged as a new principle to cope with the under-utilization of the wireless spectrum, thereby enabling more wireless-end users to acquire with more flexibility the desired quality-of-service (QoS). Many concepts have been derived from the original idea of cognitive radio [1], drawing three active research lines under the names of spectrum interweave, spectrum overlay, and spectrum underlay [2]. In this work, we focus on the underlay spectrumsharing concept as a means to allow secondary (unlicensed) users to share the same licensed spectrum band with the primary (licensed) users. This concept has the potential of enabling the secondary users to blindly access the primary system spectrum band without any prior monitoring of its occupancy. However, as far as the interference issue is concerned, the secondary user's transmit power must be kept under a certain threshold that is predetermined by the primary system, so as to legitimately maintain its QoS.

To strike a balance between adhering to the interference constraint imposed by the primary system, and ensuring additional degrees of freedom in targeting its own QoS as well, the secondary system can adopt several proactive approaches. In general, these approaches rely on a better use of the available forms of diversity in the time, frequency, and space domains. For instance, the authors in [3], [4] have suggested repetition time diversity schemes using a type-II hybrid-ARQ protocol to improve the secondary link reliability. However, the authors in [7] exploited the frequency diversity among the primary and secondary users to maximize the secondary system capacity under the interference constraint imposed by the primary system. Furthermore, multiinput multi-output (MIMO) transmissions using transmit/receive beamforming techniques have been adopted in [5], [6] to achieve high secondary system transmission rates, while minimizing the interference at the primary receiver.

Recently, it was shown that introducing relays in cognitive networks can boost the secondary outage performance especially when the deployment of multiple antennas at the source node is costly or infeasible [9]-[14], [11], [12], [13]. In these references, attention has been paid to express the outage probability of an underlay secondary system where the direct link is assisted by a set of $\mathrm{K}$ regenerative DF relaying nodes, yet the problem is still open when it comes to the proposed setup and communication model. In particular, a closed-form expression of the outage probability had been derived under complete [8] and partial [9] ICSI knowledge at the secondary transmitter while the secondary direct link undergoes a slow-fading quasi-static variations. Very recently, [13] investigated selective-and-incremental regenerative DF relaying for the secondary system under a fixed interference constraint and for two I-CSI acquisition scenarios at the level of the secondary source node. However, in the first relaying hop worst case, i.e. when no relay node or destination node has met its SNR threshold during the first hop, the source node, instead of being idle during the second hop, resets the cycle of retransmissions and proceeds with a new attempt. This implies that a significant loss in terms of spectrum efficiency may occur for low secondary system SNR ratios.

Although relevant for preserving high spectrally-efficient communications, none of the previous research work has considered selective-and-incremental regenerative decode-and-forward (DF) relaying [15] for SIMO secondary systems, where both the primary and secondary destination nodes are equipped with multiple receive antennas. Our motivating point in this contribution is therefore to study the impact of the deployment of multiple relays and receive antennas at the primary and secondary destination nodes on the overall secondary system transmission reliability and spectrum efficiency. Specifically, we are keen on providing a closed-form expression of the end-to-end outage probability for spectrally-efficient SIMO relay-aided secondary systems.

Our main results in this contribution can be summarized as follows. Firstly, we derive an outage-constrained transmit power model for the secondary system in which the transmit power of the source and relays is adapted to either a rigid interference 


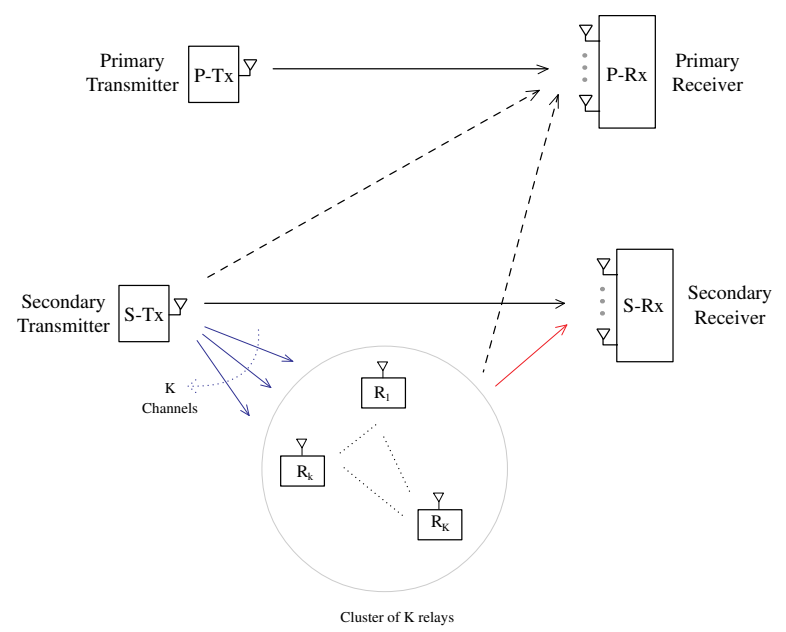

Figure 1. SIMO Relay-aided Underlay Cognitive Radio System Model.

constraint or a proportional one scaling with the primary system outage probability. Secondly, we provide the CDF of the instantaneous received SNR at each secondary receiving node during both relaying hops. Interestingly, the recursive structure of the derived expressions makes them very compact and tractable when used to subsequently derive the end-to-end secondary system outage probability in its closed-form expression. Thirdly, our analytical results are validated by simulations, while showing the positive impact of cooperative relaying and equipping the primary and secondary destination nodes with multiple antennas on the overall secondary system outage performance.

\section{FRAMEWORK DESCRIPTION}

\section{A. Proposed System Model}

Our cognitive radio setup consists of a secondary singleantenna transmitter S-Tx and an $N_{\mathrm{S}}$-antenna receiver S-Rx, both nodes are sharing the same spectrum band with a primary singleantenna transmitter P-Tx and an $N_{\mathrm{p}}$-antenna receiver P-Rx. For a spectrally-efficient secondary system transmission, a cluster $\mathcal{C}=\left\{\mathrm{R}_{\mathrm{k}}: \mathrm{k}=1, \ldots, \mathrm{K}\right\}$ of $\mathrm{K}$ single-antenna relay nodes operating in a half-duplex decode-and-forward selective relaying mode is supposed to assist S-Tx in its transmission towards S-Rx. In a cellular system, a typical communication scenario may arise in the uplink with the primary and secondary base-stations are equipped with multiple receive antennas. A schematic description of the proposed cognitive radio system model is depicted in Fig. $1^{1}$. We denote by indices $\mathrm{s}, \mathrm{p}$ and $\mathrm{k} \in\{1, \ldots, \mathrm{K}\}$ the secondary, the primary and the $\mathrm{k}^{\text {th }}$ relay nodes, respectively. Then, we refer to $\mathbf{h}_{\mathrm{pp}}, \mathbf{h}_{\mathrm{ss}}, \mathbf{h}_{\mathrm{sp}}, \mathbf{h}_{\mathrm{sk}}, \mathbf{h}_{\mathrm{ks}}$ and $\mathbf{h}_{\mathrm{kp}}$ as the frequency-flat fading mutually-independent SIMO quasi-static channel vectors connecting P-Tx with P-Rx, S-Tx with S-Rx, S-Tx with P-Rx, $\mathrm{S}-\mathrm{Tx}$ with $\mathrm{R}_{\mathrm{k}}, \mathrm{R}_{\mathrm{k}}$ with $\mathrm{S}-\mathrm{Rx}$ and $\mathrm{R}_{\mathrm{k}}$ with $\mathrm{P}-\mathrm{Rx}$, respectively. The components of $\mathbf{h}_{\mathrm{ab}} \in \mathbb{C}^{N_{\mathrm{b}} \times 1}$ with $\mathrm{a} \in\{\mathrm{s}, \mathrm{p}, \mathrm{k}\}$ and $\mathrm{b} \in\{\mathrm{s}, \mathrm{p}, \mathrm{k}\}$ are assumed to be independent identically distributed (i.i.d) and

\footnotetext{
${ }^{1}$ Herein, we consider a cognitive radio channel where the interference caused by the primary system on the secondary system receiving nodes is negligible, thereby referring to a "Z" topology of the proposed cognitive radio channel model. Investigating the impact of the primary system interference on the secondary system outage performance is left for a forthcoming contribution. Yet, if an " $\mathrm{X}$ " topology of the proposed cognitive radio channel model is adopted, our results will still hold as insightful lower bounds on the secondary system outage performance.
}

drawn from a zero-mean and $\lambda_{\mathrm{ab}}$-variance circularly symmetric complex (CSC) Gaussian distribution. Note that when index $b$ equals to $\mathrm{k}$, we have $N_{\mathrm{k}}=1$, and thus $\mathbf{h}_{\text {sk }}$ reduces to a one component channel vector. Also, since the relays are forming a cluster of $\mathrm{K}$ co-located relays, we can in fact assume that $\lambda_{\mathrm{sk}}=\lambda_{\mathrm{sr}}, \lambda_{\mathrm{kp}}=\lambda_{\mathrm{rp}}$ and $\lambda_{\mathrm{ks}}=\lambda_{\mathrm{rs}} \forall \mathrm{k} \in\{1, \ldots, \mathrm{K}\}$. For the ease of presentation, we consider the random variable change,

$$
z_{\mathrm{ab}}=\mathbf{h}_{\mathrm{ab}}^{\mathrm{H}} \mathbf{h}_{\mathrm{ab}},
$$

leading to the definition of $z_{\mathrm{ab}}$ as a random variable that is drawn from a Gamma distribution with shape and scale parameters $N_{\mathrm{b}}$ and $\lambda_{\mathrm{ab}}$, respectively.

\section{B. Secondary System Transmit Power Model}

Prior to each secondary system transmission, the transmitting nodes $\mathrm{S}$-Tx and $\mathrm{R}_{\mathrm{k}}$ for $\mathrm{k} \in\{1, \ldots, \mathrm{K}\}$ have to keep their transmit power under a maximum $\mathrm{P}_{\mathrm{s}-\max }$ and $\mathrm{P}_{\mathrm{k}-\mathrm{max}}$, respectively, while transmitting simultaneously with P-Tx. In other words, the interference caused by the secondary system at the level of the primary receiver must be limited so as not to violate the QoS of the primary system. From an outage probability perspective, $\mathrm{P}_{\mathrm{s}-\max }$ (and similarly $\mathrm{P}_{\mathrm{k} \text {-max }}$ ) can be derived as the solution to the following optimization problem,

$$
\begin{aligned}
& \operatorname{maximize} \mathrm{P}_{\mathrm{s}} \\
& \left\{\begin{array}{l}
\text { subject to } \mathrm{OP}_{\mathrm{p}} \leq \varepsilon_{\mathrm{p}} \\
\text { and } \mathrm{P}_{\mathrm{s}} \leq \overline{\mathrm{P}}_{\mathrm{s}}
\end{array}\right.
\end{aligned}
$$

where $\mathrm{OP}_{\mathrm{p}}$ denotes the outage probability of the primary system, $\varepsilon_{p}$ is an outage threshold that is defined by the primary system to maintain its QoS, and $\overline{\mathrm{P}}_{\mathrm{s}}$ is the secondary transmit power constraint. In resolving the above optimization problem, we assume that complete I-CSI about the link connecting S-Tx with $\mathrm{P}-\mathrm{Rx}$ can perfectly be estimated by S-Tx prior to sending its symbol packet to $S-R x$. The same reasoning applies to $R_{k}$ for $\mathrm{k} \in\{1, \ldots, \mathrm{K}\}$ when it transmits alongside with P-Tx.

Proposition 1: If complete I-CSI about the interference channel $\mathbf{h}_{\mathrm{sp}}$ is acquired at $\mathrm{S}-\mathrm{Tx}$, then $\mathrm{P}_{\mathrm{s}-\mathrm{max}}$ is given by,

$$
\mathrm{P}_{\mathrm{s}-\max }=\min \left\{\frac{\overline{\mathrm{P}}_{\mathrm{p}} \lambda_{\mathrm{pp}} \bar{\gamma}^{-1}\left(N_{\mathrm{p}}, \varepsilon_{\mathrm{p}}\right)-N_{0} \Phi_{\mathrm{p}}}{\Phi_{\mathrm{p}} z_{\mathrm{sp}}}, \overline{\mathrm{P}}_{\mathrm{s}}\right\},
$$

where $\Phi_{\mathrm{p}}$ is the received SNR threshold below which the primary system falls in outage. For a given real number a $\in \mathbb{R}, \bar{\gamma}^{-1}: \mathrm{x} \in$ $\mathbb{R} \rightarrow \bar{\gamma}^{-1}(\mathrm{a}, \mathrm{x}) \in \mathbb{R}$ is the inverse function of the regularized lower incomplete Gamma function $\bar{\gamma}:(\mathrm{a}, \mathrm{x}) \in \mathbb{R}^{2} \rightarrow \bar{\gamma}(\mathrm{a}, \mathrm{x}) \in \mathbb{R}[16$, Eq. 8.350.1] with respect to $\mathrm{x} . N_{0}$ denotes the variance of the additive noise at the primary receiver $\mathrm{P}-\mathrm{Rx}$ and at the secondary receiver $\mathrm{S}-\mathrm{Rx}$ as well, that is modeled as a zero-mean CSC Gaussian variable.

Proof: It simply follows from expressing the outage probability of the primary system as,

$$
\mathrm{OP}_{\mathrm{p}}=\bar{\gamma}\left(N_{\mathrm{p}}, \frac{\Phi_{\mathrm{p}}\left(\mathrm{P}_{\mathrm{s}} z_{\mathrm{sp}}+N_{0}\right)}{\overline{\mathrm{P}}_{\mathrm{p}} \lambda_{\mathrm{pp}}}\right),
$$

where the quantity $\mathrm{P}_{\mathrm{s}} z_{\mathrm{sp}}$ reflects the residual interference caused by S-Tx. Given the capability of S-Tx to acquire complete I-CSI, it can adapt its transmit power according to (2) as (3).

Remark 1. It is worth noting here that $\mathrm{P}_{\mathrm{s}-\mathrm{max}}$ should obviously be positive, i.e. the conditions $\overline{\mathrm{P}}_{\mathrm{p}} \lambda_{\mathrm{pp}} \gamma^{-1}\left(N_{\mathrm{p}}, \varepsilon_{\mathrm{p}}\right)-\Phi_{\mathrm{p}} N_{0}>0$ and $\mathrm{O}_{\max }-1>0$ must be satisfied. Interestingly, the satisfaction of both conditions depends only on the primary system settings. That 
is, the secondary system should keep silent with no transmission opportunity if the primary system settings are not favorable.

\section{PRELIMINARIES}

\section{A. Relaying Protocol}

Considering that the secondary system transmitting nodes $\mathrm{S}-\mathrm{Tx}$ and $\mathrm{R}_{\mathrm{k}}$ for $\mathrm{k} \in\{1, \ldots, \mathrm{K}\}$ are being capable of acquiring perfect ICSI about their interference channels, and therefore, transmitting with an adaptive maximum transmit power $\mathrm{P}_{\mathrm{s}-\max }$ (3) for S-Tx and similarly derived $\mathrm{P}_{\mathrm{k} \text {-max }}$ for $\mathrm{R}_{\mathrm{k}}$. During the first-hop of the proposed communication protocol, S-Tx broadcasts its symbol packet while all receiving nodes $S-R x, P-R x$, and $R_{k}$ for $k \in$ $\{1, \ldots, K\}$ listen to the transmitted symbol packet. In the case the instantaneous received signal-to-noise (SNR) ratio at S-Rx is greater than a certain threshold $\Phi_{\mathrm{s}}$, the transmitter S-Tx will move on to another symbol packet. Otherwise, one relay among the relays that have succeeded to meet their SNR thresholds will be selected to forward the received symbol packet after being correctly decoded. The ruling on the best relay selection criterion is based on the best second-hop received SNR, and is therefore given by,

$$
\mathbf{r}=\underset{\mathrm{k} \mid \mathrm{R}_{\mathrm{k}} \in \mathcal{S}}{\arg } \max \left\{\mathrm{P}_{\mathrm{k}-\max } z_{\mathrm{ks}}\right\}
$$

where $\mathrm{P}_{\mathrm{k} \text {-max }}$ is the adaptive maximum transmit power of the relay $\mathrm{R}_{\mathrm{k}}$ within $\mathcal{S}$, the subset including all relays that have met their SNR thresholds during the first-hop, thus its cardinal $|\mathcal{S}| \epsilon$ $\{0, \ldots, K\} . P_{k-\max }$ can be derived similarly to (3) as,

$$
\mathrm{P}_{\mathrm{k}-\max }=\min \left\{\frac{\overline{\mathrm{P}}_{\mathrm{p}} \lambda_{\mathrm{pp}} \gamma^{-1}\left(N_{\mathrm{p}}, \varepsilon_{\mathrm{p}}\right)-N_{0} \Phi_{\mathrm{p}}}{\Phi_{\mathrm{p}} z_{\mathrm{kp}}}, \overline{\mathrm{P}}_{\mathrm{s}}\right\} .
$$

Occasionally, the communication scheme between S-Tx and SRx may span two hops. During the first-hop, S-Rx receives the transmitted symbol packet from S-Tx, while during the secondhop, $S-R x$ receives from $R_{r}$ (index $r$ refers to the selected relay) what it successfully decoded during the first-hop. In the particular case of $|\mathcal{S}|=0$, the secondary transmitter S-Tx will retransmit during the second-hop the same symbol packet that has been transmitted during the first-hop. Therefore, at the end of the second-hop, the receiver S-Rx will sum up the two instantaneous received SNRs during both hops,

$$
\gamma_{\mathrm{ss}}^{(1)}=\min \left\{\frac{\mathrm{I}}{z_{\mathrm{sp}}^{(1)}}, \frac{\overline{\mathrm{P}}_{\mathrm{s}}}{N_{0}}\right\} z_{\mathrm{ss}}^{(1)}
$$

and either

$$
\gamma_{\mathbf{r s}}=\min \left\{\frac{\mathrm{I}}{z_{\mathbf{r p}}}, \frac{\overline{\mathrm{P}}_{\mathrm{s}}}{N_{0}}\right\} z_{\mathbf{r s}}, \text { if }|\mathcal{S}| \neq 0
$$

or

$$
\gamma_{\mathrm{ss}}^{(2)}=\min \left\{\frac{\mathrm{I}}{z_{\mathrm{sp}}^{(2)}}, \frac{\overline{\mathrm{P}}_{\mathrm{s}}}{N_{0}}\right\} z_{\mathrm{ss}}^{(2)}, \text { otherwise, }
$$

where $\mathrm{I}=\left(\overline{\mathrm{P}}_{\mathrm{p}} \lambda_{\mathrm{pp}} \gamma^{-1}\left(N_{\mathrm{p}}, \varepsilon_{\mathrm{p}}\right) /\left(\Phi_{\mathrm{p}} N_{0}\right)\right)-1$, before it decides whether the secondary system falls in outage or not. Note that the exponent $\mathrm{i} \in\{1,2\}$ in $z_{\mathrm{ss}}^{(\mathrm{i})}$ and $z_{\mathrm{sp}}^{(\mathrm{i})}$ has been added to $z_{\mathrm{ss}}$ and $z_{\mathrm{sp}}$ to differentiate between the first and the second hops since all the cognitive radio channel links experience a quasi-static change from one hop to another.

\section{B. Received SNR Statistics}

The CDF of the instantaneous received SNRs $\gamma_{\mathrm{ss}}^{(1)}, \gamma_{\mathrm{ss}}^{(2)}$, and $\gamma_{\mathrm{sk}}$ for $\mathrm{k} \in\{1, \ldots, \mathrm{K}\}$ can generally be given by,

$$
\begin{aligned}
F_{\mathrm{sb}}(z)= & P_{\mathrm{rob}}\left[\min \left\{\frac{\mathrm{I}}{z_{\mathrm{sp}}}, \frac{\overline{\mathrm{P}}_{\mathrm{s}}}{N_{0}}\right\} z_{\mathrm{sb}} \leq z\right] \\
= & \int_{0}^{\mathrm{I} N_{0} / \overline{\mathrm{P}}_{\mathrm{s}}} P_{\mathrm{rob}}\left[\frac{\overline{\mathrm{P}}_{\mathrm{s}}}{N_{0}} z_{\mathrm{sb}} \leq z\right] \frac{\mathrm{x}^{\left(N_{\mathrm{p}}-1\right)} \boldsymbol{e}^{-\mathrm{x} / \lambda_{\mathrm{sp}}}}{\lambda_{\mathrm{sp}}^{N_{\mathrm{p}}} \Gamma\left(N_{\mathrm{p}}\right)} \mathrm{dx} \\
& +\int_{\mathrm{I} N_{0} / \overline{\mathrm{P}}_{\mathrm{s}}}^{+\infty} P_{\mathrm{rob}}\left[\frac{\mathrm{I}}{\mathrm{x}} z_{\mathrm{sb}} \leq z\right] \frac{\mathrm{x}^{\left(N_{\mathrm{p}}-1\right)} e^{-\mathrm{x} / \lambda_{\mathrm{sp}}}}{\lambda_{\mathrm{sp}}^{N_{\mathrm{p}}} \Gamma\left(N_{\mathrm{p}}\right)} \mathrm{dx} \\
= & 1-\bar{\gamma}\left(N_{\mathrm{p}}, \frac{\mathrm{I} N_{0}}{\overline{\mathrm{P}}_{\mathrm{s}} \lambda_{\mathrm{sp}}}\right) \bar{\Gamma}\left(N_{\mathrm{b}}, \frac{z N_{0}}{\overline{\mathrm{P}}_{\mathrm{s}} \lambda_{\mathrm{sb}}}\right)-\frac{1}{\lambda_{\mathrm{sp}}^{N_{\mathrm{p}}} \Gamma\left(N_{\mathrm{p}}\right)} \\
& \times \sum_{\mathrm{m}=0}^{N_{\mathrm{s}}-1} \frac{\left(\frac{z}{\mathrm{I} \lambda_{\mathrm{sb}}}\right)^{\mathrm{m}}}{\mathrm{m} !} \frac{\Gamma\left(N_{\mathrm{p}}+\mathrm{m}, \frac{\mathrm{I} N_{0}}{\overline{\mathrm{P}}_{\mathrm{s}}}\left(\frac{z}{\mathrm{I} \lambda_{\mathrm{sb}}}+\frac{1}{\lambda_{\mathrm{sp}}}\right)\right)}{\left(\frac{z}{\mathrm{I} \lambda_{\mathrm{sb}}}+\frac{1}{\lambda_{\mathrm{sp}}}\right)^{N_{\mathrm{p}}+\mathrm{m}}},
\end{aligned}
$$

where index $\mathrm{b}$ should be set to $\mathrm{s}$ or $\mathrm{k}$ depending at which node $\mathrm{S}-\mathrm{Rx}$ or $\mathrm{R}_{\mathrm{k}}$ the received SNR is computed, $\Gamma(\mathrm{n}, \mathrm{x})$ is the upper incomplete Gamma function [16, Eq. 8.352.2], while $\bar{\gamma}(\mathrm{n}, \mathrm{x})=$ $\gamma(\mathrm{n}, \mathrm{x}) / \gamma(\mathrm{n})$ and $\bar{\Gamma}(\mathrm{n}, \mathrm{x})=\Gamma(\mathrm{n}, \mathrm{x}) / \Gamma(\mathrm{n})$ stand for the regularized lower and upper incomplete Gamma functions, respectively. Let us now consider that $|\mathcal{S}| \in\{1, \ldots, \mathrm{K}\}$, i.e. $|\mathcal{S}|$ relays are now eligible candidates to forward the decoded symbol packet within the second-hop.

Lemma 1: According to the proposed best relay selection method (5), the conditional CDF of the instantaneous received SNR $\gamma_{\mathbf{r s}}$ at S-Rx during the second-hop can be expressed as,

$$
\begin{aligned}
F_{\mathrm{rs}|| \mathcal{S} \mid}(z)=\sum_{\mathrm{u}=0}^{|\mathcal{S}|} e^{-\frac{z N_{\mathrm{ou}} \mathrm{u}}{\mathrm{P}_{\mathrm{s}} \lambda_{\mathrm{rs}}}} \sum_{\mathrm{v}=0}^{\mathrm{u}\left(2 N_{\mathrm{s}}+N_{\mathrm{p}}-2\right)} \mathcal{E}_{\mathrm{u}, \mathrm{v}} \\
\quad \times\left(\frac{z}{\mathrm{I} \lambda_{\mathrm{rs}}}+\frac{1}{\lambda_{\mathrm{rp}}}\right)^{\mathrm{v}-\mathrm{u}\left(N_{\mathrm{s}}+N_{\mathrm{p}}-1\right)}
\end{aligned}
$$

where $\mathcal{E}_{\mathrm{u}, \mathrm{v}}$ for $\mathrm{u} \in\{0, \ldots,|\mathcal{S}|\}$ and $\mathrm{v} \in\left\{0, \ldots, \mathrm{u}\left(2 N_{\mathrm{s}}+N_{\mathrm{p}}-2\right)\right\}$ is a multiplicative constant that can recursively be derived.

\section{OUtAgE ANALYSIS}

Using the total probability law, the end-to-end outage probability of the proposed SIMO relay-aided cognitive radio system at a given $\rho=\overline{\mathrm{P}}_{\mathrm{s}} / N_{0}$ and transmission rate $\mathcal{R}_{\mathrm{s}}$ is be given by,

$$
\operatorname{OP}_{\mathrm{s}}\left(\rho, \mathcal{R}_{\mathrm{s}}\right)=\operatorname{OP}_{\mathrm{s}}^{(1)}\left(\rho, \mathcal{R}_{\mathrm{s}}\right)+\sum_{\mathrm{k}=1}^{\mathrm{K}}\left(\begin{array}{l}
\mathrm{k} \\
\mathrm{K}
\end{array}\right) \mathrm{OP}_{\mathrm{s}, \mathrm{k}}^{(2)}\left(\rho, \mathcal{R}_{\mathrm{s}}\right),
$$

where the first term $\operatorname{OP}_{\mathrm{s}}^{(1)}\left(\rho, \mathcal{R}_{\mathrm{S}}\right)$ measures the probability of the event occurring when the secondary receiver $S$-Rx fails to meet its SNR threshold $\Phi_{\mathrm{s}}=2^{\mathcal{R}_{\mathrm{s}}}-1$ and $\dot{\Phi}_{\mathrm{s}}=2^{2 \mathcal{R}_{\mathrm{s}}}-1$ during the first and second hops, respectively, given that no relay within the cluster $\mathcal{C}$ has succeeded to meet its instantaneous SNR threshold $\dot{\Phi}_{\mathrm{S}}$ during the first-hop. Also, each term $\operatorname{OP}_{\mathrm{s}, \mathrm{k}}^{(2)}\left(\rho, \mathcal{R}_{\mathrm{s}}\right)$ of the summation in (12) corresponds to the probability of the complementary event occurring when $|\mathcal{S}|=\mathrm{k} \geq 1$, yet the $\operatorname{sum} \gamma_{\mathrm{ss}}^{(1)}+\gamma_{\mathbf{r s} \mid \mathrm{k}}$ of the received SNRs at S-Rx during the first and second hops is still inferior to $\dot{\Phi}_{\mathrm{S}}$. It is noteworthy that due to the clustered structure of the relay nodes within $\mathcal{C}$, what it matters in the derivation of $\mathrm{OP}_{\mathrm{s}, \mathrm{k}}^{(2)}\left(\rho, \mathcal{R}_{\mathrm{s}}\right)$ is the cardinal $|\mathcal{S}|=\mathrm{k}$, and not the indices of the relays belonging to the subset $\mathcal{S}$.

To derive $\mathrm{OP}_{\mathrm{S}}^{(1)}\left(\rho, \mathcal{R}_{\mathrm{S}}\right)$, we proceed by conditioning the probability (13) on $\gamma_{\mathrm{ss}}^{(2)}$ and averaging over its probability distribution 


$$
\begin{aligned}
& \operatorname{OP}_{\mathrm{s}}^{(1)}\left(\rho, \mathcal{R}_{\mathrm{s}}\right)=P_{\mathrm{rob}}\left(\gamma_{\mathrm{ss}}^{(1)}<\Phi_{\mathrm{s}}, \gamma_{\mathrm{ss}}^{(1)}+\gamma_{\mathrm{ss}}^{(2)}<\dot{\Phi}_{\mathrm{s}}, \gamma_{\mathrm{s} 1}<\dot{\Phi}_{\mathrm{s}}, \ldots, \gamma_{\mathrm{sK}}<\dot{\Phi}_{\mathrm{s}}\right) \\
& =\int_{0}^{\dot{\Phi}_{\mathrm{s}}} P_{\mathrm{rob}}\left(\gamma_{\mathrm{ss}}^{(1)}<\min \left\{\Phi_{\mathrm{s}}, \dot{\Phi}_{\mathrm{s}}-z\right\}, \gamma_{\mathrm{s} 1}<\dot{\Phi}_{\mathrm{s}}, \ldots, \gamma_{\mathrm{sK}}<\dot{\Phi}_{\mathrm{s}}\right) f_{\mathrm{ss}}(z) \mathrm{d} z, \\
& =-\int_{\dot{\Phi}_{\mathrm{s}}-\Phi_{\mathrm{s}}}^{\dot{\Phi}_{\mathrm{s}}} \frac{\mathrm{d}}{\mathrm{d} z}(\underbrace{P_{\mathrm{rob}}\left(\gamma_{\mathrm{ss}}^{(1)}<\dot{\Phi}_{\mathrm{s}}-z, \gamma_{\mathrm{s} 1}<\dot{\Phi}_{\mathrm{s}}, \ldots, \gamma_{\mathrm{sK}}<\dot{\Phi}_{\mathrm{s}}\right)}_{\mathcal{P}_{1}(z)}) F_{\mathrm{ss}}(z) \mathrm{d} z, \\
& =\mathcal{P}_{1}\left(\dot{\Phi}_{\mathrm{s}}-\Phi_{\mathrm{s}}\right)+\sum_{\mathrm{u}=0}^{N_{\mathrm{s}}-1} \frac{1}{\mathrm{u} !}\left[\bar{\gamma}\left(N_{\mathrm{p}}, \frac{\mathrm{I}}{\rho \lambda_{\mathrm{sp}}}\right)\left(\frac{1}{\rho \lambda_{\mathrm{ss}}}\right)^{\mathrm{u}} \mathrm{F}_{1}(\mathrm{u})+\frac{\Gamma\left(N_{\mathrm{p}}+\mathrm{u}\right)}{\Gamma\left(N_{\mathrm{p}}\right) \lambda_{\mathrm{sp}}^{N_{\mathrm{p}}}} \sum_{\mathrm{v}=0}^{N_{\mathrm{p}}+\mathrm{u}-1} \frac{1}{\mathrm{v} !}\left(\frac{\mathrm{I}}{\rho}\right)^{\mathrm{v}} \mathrm{F}_{2}(\mathrm{u}, \mathrm{v})\right]
\end{aligned}
$$

function, then we invoke the result via the integration by parts. As a result, (13) can be rewritten as (14) and subsequently (15) where $F_{\mathrm{ss}}($.$\left.) (resp f_{\mathrm{ss}}().\right)$ refers to the CDF (resp. PDF) of $\gamma_{\mathrm{ss}}^{(2)}$ whose expression is given by (10) before index b being replaced by $\mathrm{s}$. Once again, by conditioning $\gamma_{\mathrm{ss}}^{(1)}$ and $\gamma_{\mathrm{sk}}$ for $\mathrm{k} \in\{1, \ldots, \mathrm{K}\}$ on $z_{\mathrm{sp}}^{(1)}$, the resulting variables become mutually independent, therefore integrating over the PDF of $z_{\mathrm{sp}}^{(1)}$ becomes more tractable. This leads to writing the probability $\mathcal{P}_{1}(z)$ in (15) in a compact formula that can be expressed as,

$$
\begin{gathered}
\mathcal{P}_{1}(z)=\bar{\gamma}\left(N_{\mathrm{s}}, \frac{1}{\rho \lambda_{\mathrm{ss}}}\left(\dot{\Phi}_{\mathrm{s}}-z\right)\right) \bar{\gamma}\left(N_{\mathrm{p}}, \frac{\mathrm{I}}{\rho \lambda_{\mathrm{sp}}}\right)\left(1-e^{-\frac{\dot{\Phi}_{\mathrm{s}}}{\rho \lambda_{\mathrm{sr}}}}\right)^{\mathrm{K}} \\
+\frac{1}{\lambda_{\mathrm{sp}}^{N_{\mathrm{p}}} \Gamma\left(N_{\mathrm{p}}\right)} \sum_{\mathrm{e}=0}^{\mathrm{K}}(-1)^{\mathrm{e}}\left(\begin{array}{c}
\mathrm{K} \\
\mathrm{e}
\end{array}\right)\left[\frac{\Gamma\left(N_{\mathrm{p}}, \frac{\mathrm{I}}{\rho}\left(\frac{\dot{\Phi}_{\mathrm{s}} \mathrm{e}}{\mathrm{I} \lambda_{\mathrm{sr}}}+\frac{1}{\lambda_{\mathrm{sp}}}\right)\right)}{\left(\frac{\dot{\Phi}_{\mathrm{s}} \mathrm{e}}{\mathrm{I} \lambda_{\mathrm{sr}}}+\frac{1}{\lambda_{\mathrm{sp}}}\right)^{N_{\mathrm{p}}}}-\right. \\
\sum_{\mathrm{p}=0}^{N_{\mathrm{s}}-1} \frac{\left(\dot{\Phi}_{\mathrm{s}}-z\right)^{\mathrm{p}}}{\mathrm{p} !\left(\mathrm{I} \lambda_{\mathrm{ss}}\right)^{\mathrm{p}}} \frac{\Gamma\left(N_{\mathrm{p}}+\mathrm{p}, \frac{\mathrm{I}}{\rho}\left(\frac{\left(\dot{\Phi}_{\mathrm{s}}-z\right)}{\mathrm{I} \lambda_{\mathrm{ss}}}+\frac{\dot{\Phi}_{\mathrm{s}} \mathrm{e}}{\mathrm{I} \lambda_{\mathrm{sr}}}+\frac{1}{\lambda_{\mathrm{sp}}}\right)\right)}{\left.\left(\frac{\left(\dot{\Phi}_{\mathrm{s}}-z\right)}{\mathrm{I} \lambda_{\mathrm{ss}}}+\frac{\dot{\Phi}_{\mathrm{sp}}}{\mathrm{I} \lambda_{\mathrm{sr}}}+\frac{1}{\lambda_{\mathrm{sp}}}\right)^{N_{\mathrm{p}}+\mathrm{p}}\right]}
\end{gathered}
$$

before its derivative being replaced into (15). After some manipulations, one can rewrite the first probability as (16) where the intermediate functions $\mathcal{F}_{1}(\mathrm{u})$ and $\mathcal{F}_{2}(\mathrm{u}, \mathrm{v})$ with $\mathrm{u} \in\left\{0, \ldots, N_{\mathrm{s}}-1\right\}$ and $\mathrm{v} \in\left\{0, \ldots, N_{\mathrm{p}}+\mathrm{u}-1\right\}$ have carefully been introduced and subsequently shown to be given by,

$$
\begin{aligned}
& \mathrm{F}_{1}(\mathrm{u})=-\frac{e^{-\frac{\dot{\Phi}_{\mathrm{s}}}{\rho \lambda_{\mathrm{ss}}}}}{\left(\rho \lambda_{\mathrm{ss}}\right)^{N_{\mathrm{s}}}} \bar{\gamma}\left(N_{\mathrm{p}}, \frac{\mathrm{I}}{\rho \lambda_{\mathrm{sp}}}\right)\left(1-e^{-\frac{\dot{\Phi}_{\mathrm{s}}}{\rho \lambda_{\mathrm{sr}}}}\right)^{\mathrm{K}} \\
& \times \mathcal{I}_{0,0}^{0}\left(\mathrm{u}, N_{\mathrm{s}}-1,0,0\right)-\frac{1}{\Gamma\left(N_{\mathrm{p}}\right) \lambda_{\mathrm{sp}}^{N_{\mathrm{p}}}} \sum_{\mathrm{e}=0}^{\mathrm{K}}(-1)^{\mathrm{e}}\left(\begin{array}{c}
\mathrm{K} \\
\mathrm{e}
\end{array}\right) \\
& \times e^{-\frac{\mathrm{I}}{\rho}\left(\frac{\dot{\Phi}_{\mathrm{s}}}{\mathrm{I} \lambda_{\mathrm{ss}}}+\frac{\dot{\Phi}_{\mathrm{s}} \mathrm{e}}{\mathrm{I} \lambda_{\mathrm{sr}}}+\frac{1}{\lambda_{\mathrm{sp}}}\right)} \sum_{\mathrm{p}=0}^{N_{\mathrm{s}}-1} \frac{1}{\mathrm{p} !\left(\mathrm{I} \lambda_{\mathrm{ss}}\right)^{\mathrm{p}+1}}\left\{\left(\frac{\mathrm{I}}{\rho}\right)^{N_{\mathrm{p}}+\mathrm{p}}\right. \\
& \times \mathcal{I}_{0, \mathrm{e}}^{0}(\mathrm{u}, \mathrm{p}, 0,1)+\Gamma\left(N_{\mathrm{p}}+\mathrm{p}\right) \sum_{\mathrm{q}=0}^{N_{\mathrm{p}}+\mathrm{p}-1} \frac{\left(\frac{\mathrm{I}}{\rho}\right)^{\mathrm{q}}}{\mathrm{q} !}\left[-\mathrm{pI} \lambda_{\mathrm{ss}}\right. \\
& \times\left(\frac{\dot{\Phi}_{\mathrm{s}} \mathrm{e}}{\mathrm{I} \lambda_{\mathrm{sr}}}+\frac{1}{\lambda_{\mathrm{sp}}}\right) \mathcal{I}_{0, \mathrm{e}}^{0}\left(\mathrm{u}, \mathrm{p}-1,0, N_{\mathrm{p}}+\mathrm{p}-\mathrm{q}+1\right)+ \\
& \left.\left.N_{\mathrm{p}} \mathcal{I}_{0, \mathrm{e}}^{0}\left(\mathrm{u}, \mathrm{p}, 0, N_{\mathrm{p}}+\mathrm{p}-\mathrm{q}+1\right)\right]\right\}
\end{aligned}
$$

and

$$
\begin{aligned}
& \mathrm{F}_{2}(\mathrm{u}, \mathrm{v})=-\frac{e^{-\frac{1}{\rho}\left(\frac{\dot{\Phi}_{\mathrm{s}}}{\lambda_{\mathrm{ss}}}+\frac{\mathrm{I}}{\lambda_{\mathrm{sp}}}\right)}}{\left(\mathrm{I} \lambda_{\mathrm{ss}}\right)^{\mathrm{v}-N_{\mathrm{p}}}} \bar{\gamma}\left(N_{\mathrm{p}}, \frac{N_{0}}{\rho \lambda_{\mathrm{sp}}}\right)\left(1-e^{-\frac{\dot{\Phi}_{\mathrm{s}}}{\rho \lambda_{\mathrm{sr}}}}\right)^{\mathrm{K}} \\
& \times\left(\frac{1}{\lambda_{\mathrm{ss}}}\right)^{N_{\mathrm{s}}} \mathcal{I}_{\frac{\lambda_{\mathrm{ss}}}{\lambda_{\mathrm{sp}}}, 0}^{0}\left(\mathrm{u}, N_{\mathrm{s}}-1, N_{\mathrm{p}}+\mathrm{u}-\mathrm{v}, 0\right)-\frac{1}{\Gamma\left(N_{\mathrm{p}}\right) \lambda_{\mathrm{sp}}^{N_{\mathrm{p}}}} \\
& \times \sum_{\mathrm{e}=0}^{\mathrm{K}}(-1)^{\mathrm{e}}\left(\begin{array}{c}
\mathrm{K} \\
\mathrm{e}
\end{array}\right) e^{-\frac{\mathrm{I}}{\rho}\left(\frac{\dot{\Phi}_{\mathrm{s}}}{\mathrm{I} \lambda_{\mathrm{ss}}}+\frac{\dot{\Phi}_{\mathrm{s}} \mathrm{e}}{\mathrm{I} \lambda_{\mathrm{sr}}}+\frac{2}{\lambda_{\mathrm{sp}}}\right)} \sum_{\mathrm{p}=0}^{N_{\mathrm{s}}-1} \frac{1}{\mathrm{p} !\left(\mathrm{I} \lambda_{\mathrm{ss}}\right)^{\mathrm{p}+\mathrm{v}-N_{\mathrm{p}}+1}} \\
& \times\left\{\left(\frac{\mathrm{I}}{\rho}\right)^{N_{\mathrm{p}}+\mathrm{p}} \mathcal{I}_{\frac{\mathrm{I} \lambda_{\mathrm{ss}}}{\lambda_{\mathrm{sp}}}, \mathrm{e}}^{0}\left(\mathrm{u}, \mathrm{p}, N_{\mathrm{p}}+\mathrm{u}-\mathrm{v}, 1\right)+\Gamma\left(N_{\mathrm{p}}+\mathrm{p}\right)\right. \\
& \times \sum_{\mathrm{q}=0}^{N_{\mathrm{p}}+\mathrm{p}-1} \frac{1}{\mathrm{q} !}\left(\frac{\mathrm{I}}{\rho}\right)^{\mathrm{q}}\left[-\mathrm{pI} \lambda_{\mathrm{ss}}\left(\frac{\dot{\Phi}_{\mathrm{s}} \mathrm{e}}{\mathrm{I} \lambda_{\mathrm{sr}}}+\frac{1}{\lambda_{\mathrm{sp}}}\right)\right.
\end{aligned}
$$

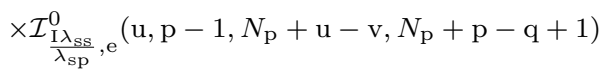

$$
\begin{aligned}
& \left.\left.+N_{\mathrm{p}} \mathcal{I}_{\frac{\mathrm{I} \lambda_{\mathrm{ss}}}{\lambda_{\mathrm{sp}}}}^{0}\left(\mathrm{e}, \mathrm{u}, \mathrm{p}, N_{\mathrm{p}}+\mathrm{u}-\mathrm{v}, N_{\mathrm{p}}+\mathrm{p}-\mathrm{q}+1\right)\right]\right\},
\end{aligned}
$$

respectively. In both functions, the integral $\mathcal{I}_{\mathrm{d}, \mathrm{e}}^{\delta}\left(\mathrm{n}_{0}, \mathrm{n}_{1}, \mathrm{n}_{2}, \mathrm{n}_{3}\right)$, where $\delta \in\{0,1\}, \mathrm{d} \in \mathbb{R}^{+*}, \mathrm{n}_{1} \in \mathbb{N} \cup\{-1\}$ and $\mathrm{n}_{0}, \mathrm{n}_{2}, \mathrm{n}_{3}, \mathrm{e} \in \mathbb{N}$, can be expressed in its general form as (20) in the top of next page. After writing $\left(\dot{\Phi}_{\mathrm{s}}-z\right)^{\mathrm{n}_{1}}$ for $\mathrm{n}_{1} \in \mathbb{N}$ in the form of a binomial expansion (in the particular case of $\mathrm{n}_{1}=-1$, the same integral resolution methodology applies), we then carry out for $v \in\left\{0, \ldots, n_{1}\right\}$ the partial fraction expansion of the following euclidean polynomial division,

$$
\begin{gathered}
\frac{z^{\mathrm{n}_{0}+\mathrm{v}}}{\left(z+\mathrm{e}_{2}\right)^{\mathrm{n}_{2}}\left(z+\mathrm{e}_{3}\right)^{\mathrm{n}_{3}}}=\boldsymbol{Q}_{\nu}(z)+\frac{\boldsymbol{R}(z)}{\left(z+\mathrm{e}_{2}\right)^{\mathrm{n}_{2}}\left(z+\mathrm{e}_{3}\right)^{\mathrm{n}_{3}}} \\
=\boldsymbol{Q}_{\nu}(z)+\sum_{\mathrm{i}=2}^{3} \sum_{\mathrm{s}=1}^{\mathrm{n}_{\mathrm{i}}} \frac{\mathcal{G}_{\mathrm{is}}}{\left(z+\mathrm{e}_{\mathrm{i}}\right)^{\mathrm{s}}},
\end{gathered}
$$

where $\boldsymbol{Q}_{\nu}(z)$ and $\boldsymbol{R}(z)$ refers to its $\nu$-degree polynomial quotient and remainder, respectively, while the coefficients $\mathcal{G}_{\text {is }}$ for $\mathrm{i} \in\{2,3\}$ and $\mathrm{s} \in\left\{1, \mathrm{n}_{\mathrm{i}}\right\}$ are derived by differentiation as,

$$
\mathcal{G}_{\text {is }}=\left.\frac{1}{\left(\mathrm{n}_{\mathrm{i}}-\mathrm{s}\right) !} \frac{\mathrm{d}^{\left(\mathrm{n}_{\mathrm{i}}-\mathrm{s}\right)}}{\mathrm{d} z}\left\{\frac{\left(z+\mathrm{e}_{\mathrm{i}}\right)^{\mathrm{n}_{\mathrm{i}}} \boldsymbol{R}(z)}{\left(z+\mathrm{e}_{2}\right)^{\mathrm{n}_{2}}\left(z+\mathrm{e}_{3}\right)^{\mathrm{n}_{3}}}\right\}\right|_{z=-\mathrm{e}_{\mathrm{i}}} .
$$

Next, expression (23) is replaced into (20). Therefore, given that $a=\delta\left(\frac{1}{\rho \lambda_{\mathrm{ss}}}-\frac{\mathrm{e}}{\rho \lambda_{\mathrm{rs}}}\right), \mathrm{e}_{2}=\mathrm{d}$, and $\mathrm{e}_{3}=-\left(\dot{\Phi}_{\mathrm{s}}+\frac{\dot{\Phi}_{\mathrm{s}} \lambda_{\mathrm{ss}} \mathrm{e}}{\lambda_{\mathrm{sr}}}+\frac{\mathrm{I} \lambda_{\mathrm{ss}}}{\lambda_{\mathrm{sp}}}\right)$, the integral $\mathcal{J}_{\mathrm{e}_{2}, \mathrm{e}_{3}}^{a}\left(\mathrm{n}_{0}, \mathrm{n}_{1}, \mathrm{n}_{2}, \mathrm{n}_{3}\right)$ can now be rewritten as,

$$
\begin{aligned}
& \mathcal{J}_{\mathrm{e}_{2}, \mathrm{e}_{3}}^{a}\left(\mathrm{n}_{0}, \mathrm{n}_{1}, \mathrm{n}_{2}, \mathrm{n}_{3}\right)=\sum_{\mathrm{v}=0}^{\mathrm{n}_{1}}\left(\begin{array}{c}
\mathrm{n}_{1} \\
\mathrm{v}
\end{array}\right)(-1)^{\mathrm{v}} \dot{\Phi}_{\mathrm{s}}^{-\mathrm{v}} \\
& \times \int_{\dot{\Phi}_{\mathrm{s}}-\Phi_{\mathrm{s}}}^{\dot{\Phi}_{\mathrm{s}}}\left[\boldsymbol{Q}_{\nu}(z)+\sum_{\mathrm{i}=2}^{3} \sum_{\mathrm{s}=1}^{\mathrm{n}_{\mathrm{i}}} \frac{\mathcal{G}_{\text {is }}}{\left(z+\mathrm{e}_{\mathrm{i}}\right)^{\mathrm{s}}}\right] \boldsymbol{e}^{a z} \mathrm{~d} z,
\end{aligned}
$$




$$
\begin{aligned}
& \mathcal{I}_{\mathrm{d}, \mathrm{e}}^{\delta}\left(\mathrm{n}_{0}, \mathrm{n}_{1}, \mathrm{n}_{2}, \mathrm{n}_{3}\right)=\frac{\dot{\Phi}_{\mathrm{s}}^{\mathrm{n}_{1}} e^{\frac{\delta \dot{\Phi}_{\mathrm{s}}}{\rho \lambda_{\mathrm{ss}}}}}{\left(-\mathrm{I} \lambda_{\mathrm{ss}}\right)^{\mathrm{n}_{3}}} \underbrace{\int_{\mathcal{J}_{\mathrm{e}_{2}, \mathrm{e}_{3}}^{a}\left(\mathrm{n}_{0}, \mathrm{n}_{1}, \mathrm{n}_{2}, \mathrm{n}_{3}\right)}^{\dot{\Phi}_{\mathrm{s}}} \frac{z^{\mathrm{n}_{0}}\left(\dot{\Phi}_{\mathrm{s}}-z\right)^{\mathrm{n}_{1}} e^{-\delta z\left(\frac{\mathrm{e}}{\rho \lambda_{\mathrm{rs}}}-\frac{1}{\rho \lambda_{\mathrm{ss}}}\right)}}{\left.(z \underbrace{\mathrm{d}}_{\mathrm{e}_{2}})^{\mathrm{n}_{2}\left(\dot{\Phi}_{\mathrm{s}}+\frac{\dot{\Phi}_{\mathrm{s}} \lambda_{\mathrm{ss}} \mathrm{e}}{\lambda_{\mathrm{sr}}}+\frac{\mathrm{I} \lambda_{\mathrm{ss}}}{\lambda_{\mathrm{sp}}}\right)}\right)^{\mathrm{n}_{3}} \mathrm{~d} z}}_{\dot{\Phi}_{\mathrm{s}}-\Phi_{\mathrm{s}}} \\
& \mathrm{OP}_{\mathrm{s}, \mathrm{k}}^{(2)}\left(\rho, \mathcal{R}_{\mathrm{s}}\right)=P_{\mathrm{rob}}\left(\gamma_{\mathrm{ss}}^{(1)}<\Phi_{\mathrm{s}}, \gamma_{\mathrm{ss}}^{(1)}+\gamma_{\mathrm{rs} \mid \mathrm{k}}<\dot{\Phi}_{\mathrm{s}}, \gamma_{\mathrm{s} 1} \geq \dot{\Phi}_{\mathrm{s}}, \ldots, \gamma_{\mathrm{sk}} \geq \dot{\Phi}_{\mathrm{s}}, \gamma_{\mathrm{sk}+1}<\dot{\Phi}_{\mathrm{s}}, \ldots, \gamma_{\mathrm{sK}}<\dot{\Phi}_{\mathrm{s}}\right) \\
& \mathrm{OP}_{\mathrm{s}, \mathrm{k}}^{(2)}\left(\rho, \mathcal{R}_{\mathrm{s}}\right)=-\int_{\dot{\Phi}_{\mathrm{s}}-\Phi_{\mathrm{s}}}^{\dot{\Phi}_{\mathrm{s}}} \frac{\mathrm{d}}{\mathrm{d} z}(\underbrace{P_{\mathrm{rob}}\left(\gamma_{\mathrm{ss}}^{(1)}<\dot{\Phi}_{\mathrm{s}}-z, \gamma_{\mathrm{s} 1} \geq \dot{\Phi}_{\mathrm{s}}, \ldots, \gamma_{\mathrm{sk}} \geq \dot{\Phi}_{\mathrm{s}}, \gamma_{\mathrm{sk}+1}<\dot{\Phi}_{\mathrm{s}}, \ldots, \gamma_{\mathrm{sK}}<\dot{\Phi}_{\mathrm{s}}\right)}_{\mathcal{P}_{2}(z)}) F_{\mathrm{rs} \mid \mathrm{k}}(z) \mathrm{d} z
\end{aligned}
$$

which, after being invoked with the help of [16, Eq. 2.323] and [17, Eq. 1.3.2.23], leads to the following closed-form expression,

$$
\begin{aligned}
& \mathcal{J}_{\mathrm{e}_{2}, \mathrm{e}_{3}}^{a}\left(\mathrm{n}_{0}, \mathrm{n}_{1}, \mathrm{n}_{2}, \mathrm{n}_{3}\right)= \\
& \left\{\begin{array}{l}
\mathcal{Q}_{\nu+1}\left(\dot{\Phi}_{\mathrm{s}}\right)-\mathcal{Q}_{\nu+1}\left(\dot{\Phi}_{\mathrm{s}}-\Phi_{\mathrm{s}}\right)+\sum_{\mathrm{i}=2}^{3}\left[\log \left(z+\mathrm{e}_{\mathrm{i}}\right)+\right. \\
\left.\frac{-1}{\left(z+\mathrm{e}_{\mathrm{i}}\right)}+\cdots+\frac{1}{\left(1-\mathrm{n}_{\mathrm{i}}\right)\left(z+\mathrm{e}_{\mathrm{i}}\right)^{\mathrm{n}_{\mathrm{i}}-1}}\right]_{\dot{\Phi}_{\mathrm{s}}-\Phi_{\mathrm{s}}}^{\dot{\Phi}_{\mathrm{s}}}, \text { if } a=0 \\
\sum_{\mathrm{v}=0}^{\mathrm{n}_{1}}\left(\begin{array}{c}
\mathrm{n}_{1} \\
\mathrm{v}
\end{array}\right)\left(-\dot{\Phi}_{\mathrm{s}}\right)^{-\mathrm{v}}\left[e^{a z} \sum_{\mathrm{k}=0}^{\nu}(-1)^{\mathrm{k}} \frac{\boldsymbol{Q}_{\nu}^{(\mathrm{k})}(z)}{a^{\mathrm{k}+1}}+\right. \\
\left.\sum_{\mathrm{i}=2}^{3} \sum_{\mathrm{s}=1}^{\mathrm{n}_{\mathrm{i}}} \frac{(-a)^{\mathrm{s}}}{a} \mathcal{G}_{\mathrm{is}_{\mathrm{s}}} e^{-a \mathrm{e}_{\mathrm{i}}} \Gamma\left(1-\mathrm{s},-a\left(z+\mathrm{e}_{\mathrm{i}}\right)\right)\right]_{\dot{\Phi}_{\mathrm{s}}-\Phi_{\mathrm{s}}}^{\dot{\Phi}_{\mathrm{s}}}, \text { if } a \neq 0
\end{array}\right.
\end{aligned}
$$

where $\mathcal{Q}_{\nu+1}(z)$ refers to the $\nu+1$-degree polynomial resulting from the indefinite integral of $\boldsymbol{Q}_{\nu}(z)$, and $\boldsymbol{Q}_{\nu}^{(\mathrm{k})}(z)$ its $\mathrm{k}^{\mathrm{th}}$ derivative. At this stage, we conclude with the derivation of the first term of the end-to-end outage probability, $\operatorname{OP}_{\mathrm{s}}^{(1)}\left(\rho, \mathcal{R}_{\mathrm{s}}\right)$. As for the second probability terms, $\mathrm{OP}_{\mathrm{s}, \mathrm{k}}^{(2)}\left(\rho, \mathcal{R}_{\mathrm{s}}\right)$ for $\mathrm{k} \in\{1, \ldots, \mathrm{K}\}$, they can similarly to (15) be expressed as (22) where $F_{\mathrm{rs} \mid \mathrm{k}}(z)$ refers to the CDF of $\gamma_{\mathbf{r s}}$ conditioned on $|\mathcal{S}|=\mathrm{k} \in\{1, \ldots, \mathrm{K}\}$ that is given by (11). After deriving $\mathcal{P}_{2}(z)$ in its closed-form expression similarly to $\mathcal{P}_{1}(z)$ in (15) and taking its derivative, the result is substituted into (22) before ending up with the following closedform expression of the second outage probability terms,

$$
\begin{gathered}
\mathrm{OP}_{\mathrm{s}, \mathrm{k}}^{(2)}\left(\rho, \mathcal{R}_{\mathrm{s}}\right)=\sum_{\mathrm{u}=0}^{\mathrm{k}} \sum_{\mathrm{v}=0}^{\mathrm{u}\left(2 N_{\mathrm{s}}+N_{\mathrm{p}}-2\right)} \mathcal{E}_{\mathrm{u}, \mathrm{v}}\left(\mathrm{I} \lambda_{\mathrm{rs}}\right)^{\mathrm{u}\left(N_{\mathrm{p}}+N_{\mathrm{s}}-1\right)-\mathrm{v}} \\
\times\left\{e^{-\frac{\dot{\Phi}_{\mathrm{s}} \mathrm{s}}{\rho \lambda_{\mathrm{sr}}} \bar{\gamma}}\left(N_{\mathrm{p}}, \frac{\mathrm{I}}{\rho \lambda_{\mathrm{sp}}}\right)\left(1-e^{-\frac{\dot{\Phi}_{\mathrm{s}}}{\rho \lambda_{\mathrm{sr}}}}\right)^{\mathrm{K}-\mathrm{k}}\left(\frac{1}{\rho \lambda_{\mathrm{ss}}}\right)^{N_{\mathrm{s}}}\right. \\
\times \mathcal{I}_{\frac{\mathrm{I} \lambda_{\mathrm{rs}}}{\lambda_{\mathrm{rp}}}, 0}^{1}\left(\mathrm{v}, N_{\mathrm{s}}-1, \mathrm{u}\left(N_{\mathrm{p}}+N_{\mathrm{s}}-1\right)-\mathrm{v}, 0\right)+\frac{1}{\lambda_{\mathrm{sp}}^{N_{\mathrm{p}}} \Gamma\left(N_{\mathrm{p}}\right)} \\
\times \sum_{\mathrm{e}=0}^{\mathrm{K}}(-1)^{\mathrm{e}}\left(\begin{array}{c}
\mathrm{K}-\mathrm{k} \\
\mathrm{e}
\end{array}\right) e^{-\frac{\mathrm{I}}{\rho}\left(\frac{\dot{\Phi}_{\mathrm{s}}}{\mathrm{I} \lambda_{\mathrm{ss}}}+\frac{\dot{\Phi}_{\mathrm{s}}(\mathrm{e}+\mathrm{k})}{\mathrm{I} \lambda_{\mathrm{sr}}}+\frac{1}{\lambda_{\mathrm{sp}}}\right)} \sum_{\mathrm{p}=0}^{N_{\mathrm{s}}-1} \frac{1}{\mathrm{p} !\left(\mathrm{I} \lambda_{\mathrm{ss}}\right)^{\mathrm{p}+1}} \\
\quad\left[\left(\frac{\mathrm{I}}{\rho}\right)^{N_{\mathrm{p}}+\mathrm{p}} \mathcal{I}_{\frac{\mathrm{I} \lambda_{\mathrm{rs}}}{\lambda_{\mathrm{rp}}}, \mathrm{e}+\mathrm{k}}^{1}\left(\mathrm{v}, \mathrm{p}, \mathrm{u}\left(N_{\mathrm{p}}+N_{\mathrm{s}}-1\right)-\mathrm{v}, 1\right)+\right. \\
\times \mathcal{I}_{\frac{\mathrm{I} \lambda_{\mathrm{rs}}, \mathrm{e}+\mathrm{k}}{\lambda_{\mathrm{rp}}}\left(\mathrm{v}, \mathrm{p}-1, \mathrm{u}\left(N_{\mathrm{p}}+N_{\mathrm{s}}-1\right)-\mathrm{v}, N_{\mathrm{p}}+\mathrm{p}-\mathrm{q}+1\right)+N_{\mathrm{p}}}^{N_{\mathrm{p}}+\mathrm{p}-1} \sum_{\mathrm{q}=0} \frac{1}{\mathrm{q} !}\left(\frac{\mathrm{I}}{\rho}\right)^{\mathrm{q}}\left(-\mathrm{pI} \lambda_{\mathrm{ss}}\left(\frac{\dot{\Phi}_{\mathrm{s}}(\mathrm{e}+\mathrm{k})}{\mathrm{I} \lambda_{\mathrm{sr}}}+\frac{1}{\lambda_{\mathrm{sp}}}\right)\right. \\
\left.\left.\left.\times \mathcal{I}_{\frac{\mathrm{I} \lambda_{\mathrm{rs}}}{\lambda_{\mathrm{rp}}}, \mathrm{e}+\mathrm{k}}^{1}\left(\mathrm{v}, \mathrm{p}-1, \mathrm{u}\left(N_{\mathrm{p}}+N_{\mathrm{s}}-1\right)-\mathrm{v}, N_{\mathrm{p}}+\mathrm{p}-\mathrm{q}+1\right)\right)\right]\right\} .
\end{gathered}
$$

As evidence of both expressions (16) and (27), the derived closedform expression of the end-to-end outage probability (12) is valid for any value $\mathrm{K}, \overline{\mathrm{P}}_{\mathrm{s}}, N_{\mathrm{s}}$, and channel statistics $\lambda_{\mathrm{ab}}$ with $\mathrm{a} \in\{\mathrm{s}, \mathrm{p}, \mathrm{k}\}$ and $\mathrm{b} \in\{\mathrm{s}, \mathrm{p}, \mathrm{k}\}$. Consequently, it can be used to evaluate the outage performance of a wide range of SIMO relayaided underlay cognitive radio system models.

\section{Simulation Results}

\section{A. Network Geometry}

Due to large-scale variations of the cognitive radio channel, the second-order statistics of the link connecting each pair of nodes in our system can be expressed as $\lambda_{\mathrm{ab}}=d_{\mathrm{ab}}^{-\kappa}$ where $d_{\mathrm{ab}}$ refers to the local distance between the transmitting and receiving nodes whose indices are $\mathrm{a} \in\{\mathrm{s}, \mathrm{p}, \mathrm{k}\}$ and $\mathrm{b} \in\{\mathrm{s}, \mathrm{p}, \mathrm{k}\}$, respectively. $\kappa$ denotes the path-loss exponent, and is set to 4 as a practical value. For the sake of simplicity, we consider, without loss of generality, a linear network configuration in which the cluster $\mathcal{C}$ is located between the secondary transmitter S-Tx and the secondary receiver S-Rx such that $d_{\mathrm{sr}}+d_{\mathrm{rs}}=d_{\mathrm{ss}}=d_{\mathrm{sp}}=d_{\mathrm{pp}}=1$. Despite of the relay location between S-Tx and S-Rx, to obtain a good outage performance results one may suggest to choose the farthest possible position of the cluster $\mathcal{C}$ from $\mathrm{P}-\mathrm{Rx}$ so as not to severely interfere with its own reception. In fact, if $0<d_{\mathrm{rp}}<1$, we can choose $d_{\mathrm{rp}}=0.9$ as a comprehensive value. After carrying several analytical simulations, we realized that the optimum cluster location leading to the minimum secondary system outage performance is centered around the conventional position $d_{\mathrm{sr}}=0.5$. In the sequel, we keep these cognitive radio system settings unchanged, and conduct our analytical and simulation results analysis for a quiet strict primary system outage constraint $\varepsilon_{\mathrm{p}}=0.001$.

\section{B. Analytical and Numerical Results}

In Fig. 2, the end-to-end outage probability expression (12) of the proposed SIMO relay-aided cognitive radio system are depicted for several secondary system settings. We have considered that $\overline{\mathrm{P}}_{\mathrm{p}}$ is fixed at the value of $20 \mathrm{~dB}$, and consequently independent of $\overline{\mathrm{P}}_{\mathrm{s}}$ which is growing up in the $\mathrm{x}$-axis. In this case, the end-to-end outage probability saturates at its floor values. Clearly, the outage floor values decrease with an increasing number of $N_{\mathrm{s}}$ and K. For instance, from a system configuration where $N_{\mathrm{s}}=2$ and $\mathrm{K}=2$ to another one where $N_{\mathrm{s}}=4$ and $\mathrm{K}=4$, the end-to-end outage probability decreases from the value 


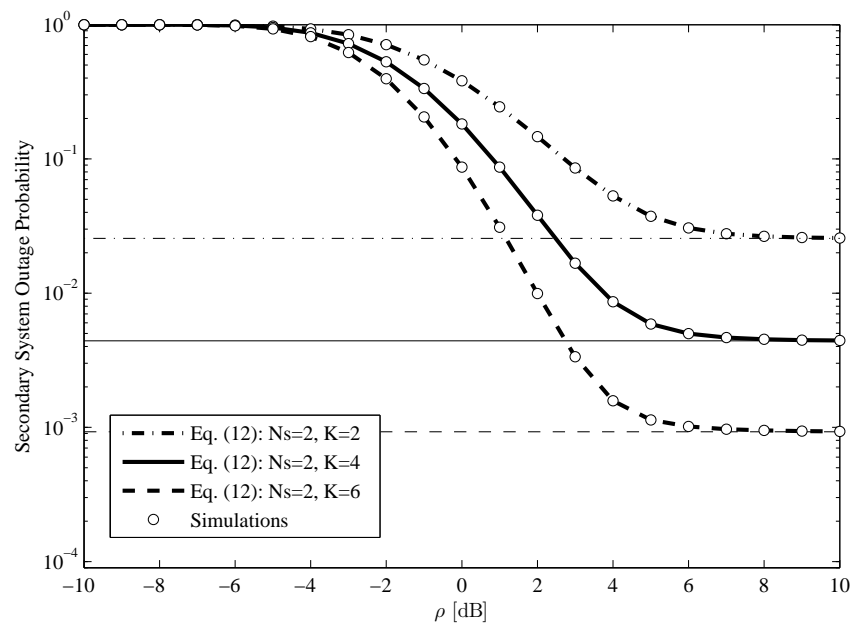

Figure 2. The analytical expressions of the derived secondary system outage probability are compared to those found by Monte Carlo simulations, for $\overline{\mathrm{P}}_{\mathrm{p}}=$ $20 \mathrm{~dB}, N_{\mathrm{p}}=4$, and $\mathcal{R}_{\mathrm{s}}=\mathcal{R}_{\mathrm{p}}=2$ bits $/ \mathrm{s} / \mathrm{Hz}$.

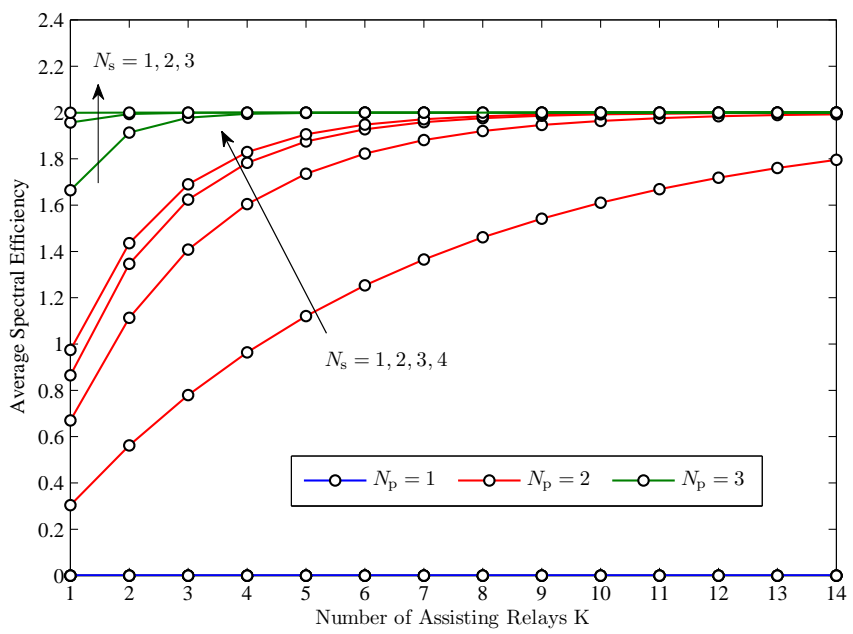

Figure 3. Secondary system average spectral efficiency in bits/s/Hz vs the number of the assisting relays $\mathrm{K}$, for $\overline{\mathrm{P}}_{\mathrm{p}}=20 \mathrm{~dB}$, and $\mathcal{R}_{\mathrm{s}}=\mathcal{R}_{\mathrm{p}}=2 \mathrm{bits} / \mathrm{s} / \mathrm{Hz}$.

of $3.10^{-2}$ to $3 \cdot 10^{-4}$. This suggests that in an underlay cognitive radio system, the secondary transmitter may greatly gain from the assistance of relays and the deployment of multiple antennas at the secondary and primary receivers. The same remarks hold true even when $\overline{\mathrm{P}}_{\mathrm{p}}<20 \mathrm{~dB}$ and $N_{\mathrm{p}}<4$, but with the curves will be translating up.

Intuitively, when multiple receive antennas $N_{\mathrm{p}}$ are deployed at the level of the primary receiver, the interference constraint imposed on the secondary system transmit power will considerably be relaxed giving more degrees of freedom to the secondary system in acquiring a better outage performance. As observed in Fig. 3, no opportunity in average for the secondary system to transmit alongside with the primary system in the case of $N_{\mathrm{p}}=1$. On the contrary, the secondary system average spectral efficiency tends to quickly improve when $N_{\mathrm{p}}$ increases. Therefore, deploying more than one antenna at the primary system receiver is viewed as an obligation to attain spectrally-efficient secondary transmissions. Also, the same figure shows the substantial gains that can be reaped via cooperative relaying and the deployment of multiple antennas at the primary and secondary receivers. Importantly, with only three receive antennas at the primary and secondary destination nodes and a cluster of four relays, the maximum average spectral efficiency of $2 \mathrm{bits} / \mathrm{Hz} / \mathrm{seconds}$ is already achieved.

\section{CONCLUSION}

In this contribution, we have derived expressions of the outage probability for SIMO relay-aided secondary systems under a strict primary system outage constraint. Finally, the analytical results are validated by simulations while revealing that cooperative relaying and the deployment of multiple antennas at the primary and secondary receivers are two gainful facts that can boost the outage performance and considerably enhance the spectral efficiency of a cognitive radio system in a spectrum sharing underlay context.

\section{REFERENCES}

[1] J. Mitola and G. Q. Maguire, "Cognitive radio: Making software radios more personal," IEEE Pers. Commun., vol. 6, no. 4, pp. 13-18, Aug. 1999.

[2] A. Glodsmith, S. A. Jafar, I. Marić, and S. Srinivasa, "Breaking spectrum gridlock with cognitive radios: An information theoretic perspective," Proceedings of the IEEE, vol. 97, no. 5, pp. 894-914, May 2009.

[3] B. Makki, T. Eriksson, "On the average Rate of HARQ-based Quasi-Static Spectrum Sharing Networks," IEEE Trans. on Wireless Communications, vol. 11, no. 1, pp. 65-77, Jan. 2012.

[4] L. Musavian, T. Le-Ngoc, "Cross-Layer Design for Cognitive Radios with Joint AMC and ARQ Under Delay QoS Constraint," in Proc. International Wireless Communications and Mobile Computing Conference, Cyprus, Turkey, August 2012.

[5] R. Zhang and Y.-C. Liang, "Exploiting multiantennas for opportunistic spectrum sharing in cognitive radio networks," IEEE Journal on Selected Topics in Signal Processing, vol. 2, no. 1, pp. 88-102, Feb. 2008.

[6] H. Wang, J. Lee, S. Kim, and D. Hong, "Capacity enhancement of secondary links through spatial diversity in spectrum sharing," IEEE Trans. on Wireless Communications, vol. 9, no. 2, pp. 494-499, Feb. 2010.

[7] G. Zhao, C. Yang, G. Y. Li, D. Li, and A. C. K. Soong, "Power and channel allocation for cooperative relay in cognitive radio networks," IEEE Journal on Selected Topics in Signal Processing, vol. 5, no. 1, pp. 151-159, Feb. 2011.

[8] Z. El-Moutaouakkil, K. Tourki, K. A. Qaraqe, S. Saoudi, "Exact Outage Probability Analysis for Relay-Aided Underlay Cognitive Communications," in Proc. IEEE Vehicular Technology Conference Fall, Québec City, Canada, Sep. 2012.

[9] Y. Zou, J. Zhu, B. Zheng, and Y.-D. Yao, "An adaptive cooperation diversity scheme with best-relay selection in cognitive radio networks," IEEE Trans. on Signal Processing, vol. 58, no. 10, pp. 5438 -5445, 2010.

[10] L. Luo, P. Zhang, G. Zhang, and J. Qin, "Outage performance for cognitive relay networks with underlay spectrum sharing," IEEE Communications Letters, vol. 15, no. 7, pp. 710-712, Jul. 2011.

[11] J. Si, Z. Li, X. Chen, B. Hao, and Z. Liu, "On the performance of cognitive relay networks under primary user's outage constraint," IEEE Communications Letters, vol. 15, no. 4, pp. 422-424, Apr. 2011.

[12] Z. Yan, X. Zhang, and W. Wang, "Exact Outage Performance of Cognitive Relay Networks with Maximum Transmit Power Limits," IEEE Communications Letters, vol. 15, no. 12, pp. 1317-1319, Dec. 2011.

[13] K. Tourki, K. A. Qaraqe, and M. Alouini, "Outage Analysis for Underlay Cognitive Networks Using Incremental Regenerative Relaying," IEEE Trans. on Vehicular Technology, vol. 62, no. 2, pp. 721-734, Feb. 2013.

[14] K. J. Kim, T. Q. Duong, H. V. Poor, "Outage Probability of Single-Carrier Cooperative Spectrum Sharing Systems with Decode-and-Forward Relaying and Selection Combining," IEEE Trans. on Communications, vol. 12, no. 2, pp. 806-817, Feb. 2013.

[15] R. Tannious and A. Nosratinia, "Spectrally-Efficient Relay Selection with Limited Feedback," IEEE Journal on Selected Areas in Communications, vol. 26 , no. 8, pp. 1419-1428, Oct 2008.

[16] I. S. Gradshteyn and I. M. Ryzhik, Table of Integrals, Series, and Products, 7th ed. San Diego, CA: Academic, 2007.

[17] A. P. Prudnikov, Yu. A. Brychkov, O. I. Marichev, Integrals and Series, vol. 1, Gordon and Breach Science Publishers, 1986. 